\title{
Short-loop algorithm for quantum Monte Carlo simulations
}

\author{
Ying-Jer Kao* \\ Department of Physics and Center for Theoretical Sciences, National Taiwan University, Taipei 106, Taiwan \\ Roger G. Melko ${ }^{\dagger}$ \\ Department of Physics and Astronomy, University of Waterloo, Ontario, Canada N2L $3 G 1$ \\ and Materials Science and Technology Division, Oak Ridge National Laboratory, Oak Ridge, Tennessee 37831, USA \\ (Received 11 September 2007; revised manuscript received 12 December 2007; published 25 March 2008)

\begin{abstract}
We present an algorithmic framework for a variant of the quantum Monte Carlo operator-loop algorithm,
\end{abstract} \\ where nonlocal cluster updates are constructed in a way that makes each individual loop smaller. The algorithm \\ is designed to increase simulation efficiency in cases where conventional loops become very large, do not close \\ altogether, or otherwise behave poorly. We demonstrate and characterize some aspects of the short loop on a \\ square lattice spin-1/2 XXZ model where, remarkably, a significant increase in simulation efficiency is ob- \\ served in some parameter regimes. The simplicity of the model provides a prototype for the use of short loops \\ on more complicated quantum systems.
}

DOI: 10.1103/PhysRevE.77.036708

PACS number(s): $02.70 . S s, 75.10 . J m$

\section{INTRODUCTION}

Quantum Monte Carlo (QMC) simulations [1,2] comprise arguably the most powerful set of methods for analyzing strongly interacting models in quantum many-body physics. Breakthroughs in QMC methodology over the last decade have enabled the study of simulation cells of unsurpassed finite size, many capable of simulating millions of quantum species for simple models. Traditionally, large system sizes were coveted to enable finite-size scaling to the thermodynamic limit, something that remains important for the study of quantum ground states and critical phenomena, where unconventional or nonmonotonic scaling is sometimes observed [3]. However, recent interest in nanoscale quantum systems, as well as ultracold atoms trapped in optical lattices, has provided a situation where QMC methods are able to approach realistic experimental system sizes [4]. The work on algorithmic advances therefore continues at a rapid pace.

Besides the infamous sign problem $[5,6]$, which precludes the simulation of many fermionic and frustrated magnetic systems, the largest general obstacle for QMC methods are algorithm freezing, critical slowing down, or other phenomena perhaps best summarized as "loss of ergodicity." These can result in problems ranging from a slight loss of efficiency (requiring longer Monte Carlo runs to reach a desired level of statistical accuracy), to serious issues such as complete nonergodicity in some parameter regimes, leading to the obscuration of all interesting physics in the model. For example, an inability to accurately measure a subset of estimators (in particular off-diagonal quantities) is a drawback of some classes of simple "local" QMC updates [7].

Perhaps the most important algorithmic breakthrough in QMC technology was the introduction of the loop algorithm by Evertz, Lana, and Marcu [8]. Until that time, the QMC sampling procedure proceeded via local updates, roughly

\footnotetext{
*yjkao@phys.ntu.edu.tw

†rgmelko@science.uwaterloo.ca
}

analogous to single spin flips in a simple Monte Carlo simulation of a classical Ising model [7]. The loop algorithm, analogous to a Wolff or Swenson-Wang cluster (or global) update, solved ergodicity problems related to sampling in a grand-canonical framework, and also facilitated the measurement of some off-diagonal quantities. Originally formulated in a discrete world-line framework, the algorithm has been continually refined and advanced, and is widely used in all modern QMC frameworks, for example, in continuous world-line methods (including worm algorithm variants $[9,10]$ ) and the stochastic series expansion (SSE) framework, which employs the "operator" [11] or "directed"-loop variants [12].

The common feature of all QMC loop algorithms is the creation of a defect or singular point (or in the case of the worm algorithm, two points) which propagates through the simulation cell updating the QMC representation of the Hamiltonian or partition function (i.e., the world-line configuration, or the basis state and operator list). This defect is typically resolved when it encounters its starting point (or another propagating defect), forming a single closed loop. Loops formed in this way may then be used in a variety of single-cluster or multicluster sampling schemes $[7,13]$. In the following, we will call such an algorithm, where the closing condition of the loop is that its "head" meets its "tail," a conventional or long loop.

In classical Monte Carlo methods, the prototypical analogy of the above algorithm was first introduced for the problem of proton distribution in ice water [14], and later extended to Monte Carlo simulations of other vertex and ice models [15]. This classical loop algorithm effectively allows targeted updates in a reduced manifold of low-energy vertex states. The original classical loop is the long loop, as described above (see also Ref. [16]); however, a variation that involves loops of shorter length has been shown to perform more efficiently in a large number of cases, and has become widely adopted $[15,17]$. This variation became known as the short-loop algorithm, and as its name implies, involves creating loops of much smaller total length. A key reason for the increase in efficiency observed with short loops appears to be 


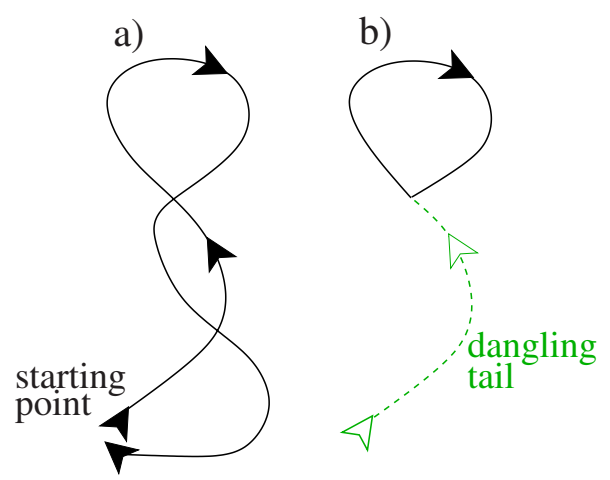

FIG. 1. (Color online) Schematic comparison of a long loop (a) versus a short loop (b). In (a), the loop defect propagates until it encounters its own starting point. In (b), the loop defect propagates only until it encounters its own path. The dangling tail (dashed green line) must be removed.

a respite from the tendency of long loops to grow in proportion to the size of the simulation cell, which in some cases can result in excessively long updates and a delay in defect resolution [17]. Additionally, short loops do not have the capacity to retrace multiple paths through the same region of configuration space, avoiding the wasted computational overhead that often can occur in long-loop algorithms.

Conceptually, such short loops are distinguished from the long-loop construct based simply on the closing or resolution condition of the loop's head or defect. Namely, a short loop closes not only if the defect encounters its own starting point (i.e., the head meets the tail), but also if it encounters any other previous point of the loop body. Short loops are also differentiated by the resulting dangling tail of propagated defects, which must be removed from the loop structure before the Monte Carlo update can continue (see Fig. 1).

From this description, the classical definition [15,17] of the short-loop algorithm can be adapted to the case of an operator-loop algorithm in a $d+1$ quantum simulation cell. In this paper, we provide a detailed description of the short-loop algorithm in a full QMC framework. We note that short loops may be formulated in any of the aforementioned QMC algorithmic flavors; in the next section we choose to introduce them in the popular and simple SSE QMC paradigm $[11,12,18,19]$. We are particularly motivated by the question of whether the large efficiency gains enjoyed by short loops in classical Monte Carlo simulations of vertex models will translate over to the QMC arena. In Sec. IV, we attempt to answer this question with concrete autocorrelation measurements on the simple demonstrative case of the twodimensional (2D) $S=1 / 2 X X Z$ model. We conclude the paper with a short discussion of several advantages and disadvantages of the short-loop algorithm, and possible adaptations of it to more complicated quantum models in the future.

\section{LOOP ALGORITHMS IN THE STOCHASTIC SERIES EXPANSION FRAMEWORK}

The SSE decomposition of a quantum Hamiltonian on a $d$-dimensional lattice proceeds via the expansion of the finite-temperature partition function $[12,18]$,

$$
Z=\sum_{\alpha} \sum_{n=0}^{\infty} \sum_{S_{n}} \frac{(-\beta)^{n}}{n !}\left\langle\alpha\left|\prod_{i=1}^{n} H_{t_{i}, b_{i}}\right| \alpha\right\rangle .
$$

Here, the sum over $S_{n}$ represents a sampling of an operatorindex sequence (defined below), performed via a Metropolis Monte Carlo procedure. In $Z$, a quantum Hamiltonian is typically written as a sum of elementary interactions,

$$
H=-\sum_{t} \sum_{b} H_{t, b}
$$

where in a chosen basis $\{|\alpha\rangle\}$ (e.g., the standard $S^{z}$ basis) the operators satisfy $H_{t, b}|\alpha\rangle \sim\left|\alpha^{\prime}\right\rangle$, and $|\alpha\rangle$ and $\left|\alpha^{\prime}\right\rangle$ are both basis states. The index $t$ refers to the operator types (various kinetic and potential terms), while $b$ is the lattice unit over which the interactions are summed (e.g., a nearest-neighbor bond). The operator-index sequence is hence represented as $S_{n}=\left[t_{1}, b_{1}\right] \cdots\left[t_{n}, b_{n}\right]$, where $n$ is the expansion order. Typically, the size of the operator-index sequence is set to some constant $M>n$ (since $n$ fluctuates), and the operator-index list is filled in with unit or identity operators, represented in $S_{M}$ as $[0,0]$.

For concreteness, we will consider the paradigmatic spin$1 / 2 X X Z$ model,

$$
H=J \sum_{\langle i, j\rangle}\left(S_{i}^{x} S_{j}^{x}+S_{i}^{y} S_{j}^{y}+\Delta S_{i}^{z} S_{j}^{z}\right)-h \sum_{i} S_{i}^{z} .
$$

A standard SSE algorithm for this Hamiltonian is laid out in detail in Ref. [12], and we refer the reader to that work as we make frequent reference to it in the upcoming discussion. In particular, the square lattice decomposition [Eq. (2)] for this Hamiltonian results in two bond terms,

$$
\begin{gathered}
H_{1, b} / J=C-\Delta S_{i}^{z} S_{j}^{z}+\frac{h}{4 J}\left(S_{i}^{z}+S_{j}^{z}\right), \\
H_{2, b} / J=\frac{1}{2}\left(S_{i}^{+} S_{j}^{-}+S_{i}^{-} S_{j}^{+}\right),
\end{gathered}
$$

where the constant $C$ is defined as necessary to make $H_{1, b}$ $>0$, hence avoiding the sign problem.

There are two standard (nontrivial) updates for SSE simulations of typical Hamiltonians. The first is the diagonal update, designed to perform substitutions $[0,0] \leftrightarrow[1, b]$, changing the expansion order $n$. The second update, of interest to us, is the operator loop update, which accomplishes substitutions within and between operator-list elements $[1, b]$ and $[2, b]$, keeping $n$ fixed but effectively sampling off-diagonal operators. The operator loop is performed in a linked list of vertices, an abstraction of the propagation of the basis state $|\alpha\rangle$ by $S_{M}$ in the $d+1$-dimensional simulation cell [12]. The linked list is defined graphically by single operators propagating a unit's (bond's) basis state at some given expansion step (see Fig. 2). In the $S=1 / 2 X X Z$ model, there are six allowed vertices resulting from six nonzero matrix elements [see Eq. (18) of Ref. [12]].

In the conventional long-loop SSE algorithm, a vertex is updated by a propagating defect. The defect propagates along the linked list and, upon meeting its own starting point (i.e., when the head meets the tail), forms a closed loop. 


$$
\left\langle\uparrow \downarrow\left|H_{2, b}\right| \downarrow \uparrow\right\rangle=\frac{\bigcirc}{\bigcirc} \bigcirc
$$

FIG. 2. A vertex as a graphical representation of a bond matrix element. Filled circles represent spin $+1 / 2$, open represent spin $-1 / 2$. For the $X X Z$ model [Eq. (3)] this vertex has weight $J / 2$ [12].

Typically, the starting point of the loop is chosen randomly from the linked vertex list. During the propagation, the defect enters a vertex simply by following a link from the "exit leg" of the previously visited vertex. An exit leg is typically chosen by a Metropolis Monte Carlo procedure: For example, a simple heat-bath scheme where the probability of exiting along any given vertex leg is proportional to the weight of the resulting matrix element. A particularly efficient way to choose these exit probabilities in the SSE is to use the directed-loop equations, detailed in Ref. [12] however, the form of the loop algorithm (long or short) is independent of the choice of exit probabilities.

Once closed, a long loop satisfies detailed balance and, in effect, the visited vertex legs may be flipped with probability 1 -hence its relationship to the classical Wolff cluster algorithm [13]. In practice, one need not store the loop path at all, as updating of the vertex legs occurs in real time as the defect propagates. Once closed, the vertices visited by the loop are already affected (i.e., flipped) and one must simply update the stored global basis state $|\alpha\rangle$ and operator-index sequence $S_{M}$. Note that this update typically occurs after a significant number of loops have been preformed-this number is discussed much more below.

As alluded to above, one difficulty encountered in this loop algorithm for some parameter regimes of certain Hamiltonians [not necessarily Eq. (3)] is that loops can become very long before they close, or sometimes in extreme cases do not close at all $[20,21]$. The standard practice to combat this is to impose some maximum loop length

$$
\ell_{\text {loop }}^{\max }=c_{o} n
$$

( $c_{o}$ is some constant), upon reaching which loop construction is terminated. Here, loop length may be measured, for example, in the number of vertex legs traversed per algorithm iteration (typically two). In the case of termination, detailed balance is preserved by disregarding updates attained by the loop, and keeping the previous Monte Carlo steps $|\alpha\rangle$ and $S_{M}$ [12]. Unfortunately, the algorithm overhead (i.e., CPU time) used in constructing the aborted loop or set of loops is lost in this case.

These examples serve to further motivate the development of a loop algorithm that does not suffer from such drawbacks. One solution, in analogy to the classical shortloop algorithms discussed in the preceding section, is a quantum short-loop variant of the conventional SSE operator loop. In the next section, we discuss details of the quantum short-loop algorithm, including the closing condition, handling of bounce processes, and tail removal.

\section{SHORT-LOOP ALGORITHM}

\section{A. Overview}

At first glance, the definition of the short-loop algorithm in the SSE is quite simple. Begin by propagating a loop
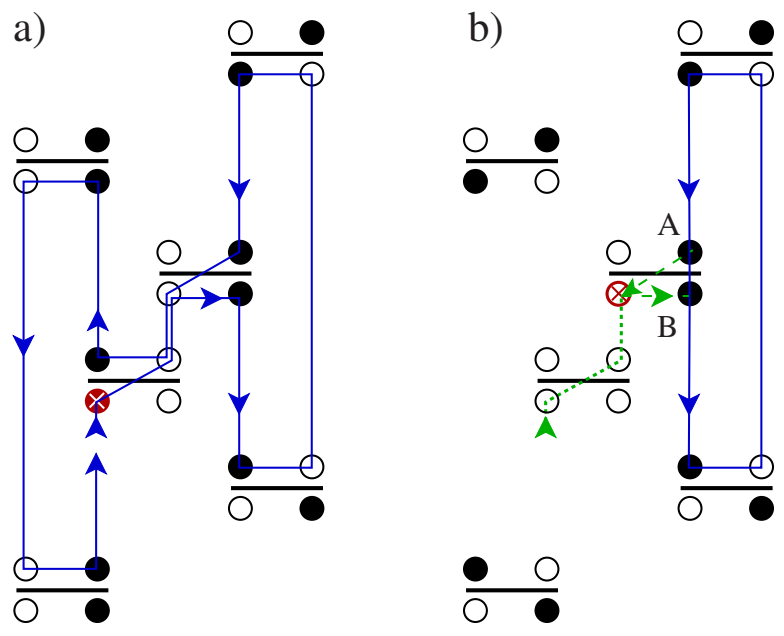

FIG. 3. (Color online) A linked list of six vertices, with operator loops and final (i.e., flipped) basis state spins. For clarity, links are not illustrated, but occur as vertical lines connecting vertex legs [12]. (a) A long loop, which propagates around the linked list until it encounters its own original starting vertex leg (filled red with a cross). (b) The same loop construction, if governed by short-loop rules, would propagate around the linked list until it encounters a previously visited vertex leg which was also an in leg (open red with a cross). The loop closes in the vertex containing this leg, by connecting the remaining visited sites $(A$ and $B)$. The beginning portion of the loop propagation, the dangling tail (dotted green line), is removed and the associated vertex legs are not flipped.

defect as one would normally do for the long operator loop, starting from a random vertex leg. In the event where the propagating defect encounters a vertex leg where it has previously been, terminate the loop algorithm. The segment of the path created by the defect that does not form the loop is the dangling tail (see Fig. 1), and must be removed or reverted back to its original state. Also, a consequence of the need to remove this tail is the requirement to store the loop path created by the propagating defect-something that is not needed in the conventional long-loop algorithm.

Consider the important closing condition of the short-loop algorithm in more detail. It turns out that, unlike the classical case, the simplified criterion mentioned above (the loop closes upon encountering any previously visited vertex leg) is insufficient for the QMC case, since a quantum operator vertex is involved. To facilitate closing of the quantum short loop, the terminating leg should have been, upon its original visit, an in leg (see Fig. 3); if the propagating defect encounters a previously visited out leg, the loop creation algorithm should continue unabated. An attempt to close the short loop at an out leg would result in an unresolvable defect, where removing the dangling tail becomes impossible without destroying the loop itself. Once the terminating leg is chosen, its spin is not flipped, and the loop is closed at that vertex using the remaining two visited legs, finally resolving the propagating defect. Assuming that one has flipped spins associated with vertex legs during the propagation of the defect, starting from the terminating vertex leg, flip back all spins and update the associated vertices on the dangling tail, until the initial starting point is reached. Since this tail re- 
moval occurs deterministically, detailed balance remains satisfied for the short-loop algorithm. The short loop is now complete, and the usual progression of the SSE Monte Carlo algorithm (i.e., more loop updates, diagonal updates, or measurements) may proceed.

A comparison between two long and short loops is illustrated in Fig. 3. Even in this simple case, several key factors that determine loop efficiency are apparent. First, the short loop is obviously much smaller (in the total number of vertices visited) than the long loop, which served as the original motivation for designing this algorithm. Further, by inspecting the center of Fig. 3(a), it is apparent that two vertex legs have been visited two times in this illustration. Processes such as this ("retracing") may have a negative impact on long-loop performance, since the computational effort associated with propagating the defect through these vertex legs may ultimately result in no flipped basis spins. In this way, it is apparent that a long loop could correspond to constructing separate smaller loops and flipping them all together. In contrast, the same smaller loops, if constructed with several short-loop updates, would flip the loops independently. Issues such as these may also result in slight improvements in efficiency when using the short-loop algorithm.

Any gains of this sort must, however, be weighed against the computational overhead associated with storing the short loop, resolving the propagating defect in the terminating vertex, and removing the dangling tail. The process is illustrated in Fig. 3(b), where the dangling tail involves three vertex legs (two of which must be reflipped). In addition to this computational overhead, additional data structures are required in the formation of the short-loop algorithm to store the loop path in memory to allow for the removal of the tail. Since the additional CPU time and memory burdens may conceivably negate any efficiency improvements gained on the long loop, the simple arguments associated with Fig. 3 are likely not sufficient to draw quantitative conclusions of short-loop performance-this is left to Sec. IV where we discuss autocorrelation results. Before addressing this, we proceed with several more key details to note when implementing the short-loop algorithm in a practical QMC code.

\section{B. Short loops in the presence of bounce processes}

In the preceding discussion, one important complicating factor was purposely neglected: The handling of so-called bounce processes in the formation of the operator loops. Bounce processes (see also Ref. [12]) are defined as the case where a propagating loop defect, upon encountering any given vertex, chooses (by way of the specific Hamiltonian and algorithmic probability tables) its out leg to be the same as its in leg, thereby starting on a path which retraces, for some distance at least, the loop back along its path of previously visited vertex legs.

Bounce processes are known to be the most serious detriment to the efficiency of the loop update in the SSE [12] (although they are perhaps not the only detrimental process [22]). Advanced methods to construct the QMC probability tables governing loop propagation, such as the directed-loop weights $[12,22,23]$, combat this problem by minimizing the
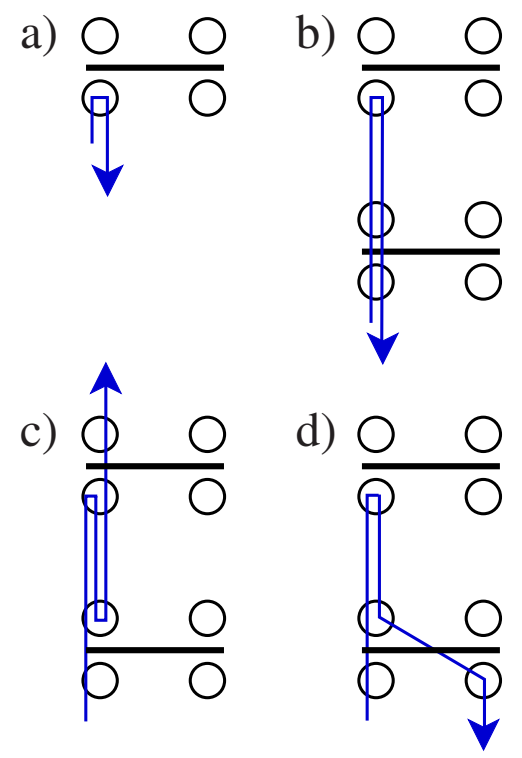

FIG. 4. (Color online) (a) A bounce process which (b) continues to retrace the loop path, (c) rebounces to continue a new path, (d) branches to continue a new path.

weight of bounce processes when possible. However, it is common to find many physically interesting models where bounce processes cannot be avoided. As such, any practical implementation of a short-loop algorithm must be able to take bounces into account.

The simple short-loop algorithm described in Sec. III A requires several modifications, which are essentially conditions to ensure that the loop does not terminate prematurely if it encounters its own path due to a bounce process. Recall that, in order to remove the dangling tail upon termination, the short loop requires storage of the loop propagation path. Considering the possibility of bounce processes, it becomes obvious that a modified stack (last in, first out) is the appropriate data structure with which to store the loop propagation. Vertex legs which are part of a new path should be pushed onto the top of the stack, while bounces or retraced vertex legs should be popped off the top. More specifically, several cases should be considered (see Fig. 4). First, in the simple case of a first bounce, easily identifiable since the in leg is identical to the out leg, the first bounce vertex leg is not added to the stack [Fig. 4(a)]. If the bounce continues along the previously visited path [Fig. 4(b)], previously visited vertex legs are popped off the top of the stack. New legs are added to the stack when the path deviates from the previously visited path, as in the cases shown in Figs. 4(c) and 4(d). Note that only these last two cases, where the propagating defect begins tracing a new path, should have the option of closing the short loop.

After the short loop eventually closes, this stack data structure is accessed from the terminating vertex leg and retraced to the bottom of the stack to remove the dangling tail (see Fig. 3). With bounce processes pushed and popped correctly during defect propagation, the algorithmic overhead involved with removing the tail can be reduced considerably.

With these considerations, the problem of implementing the short-loop algorithm is essentially a coding procedure- 

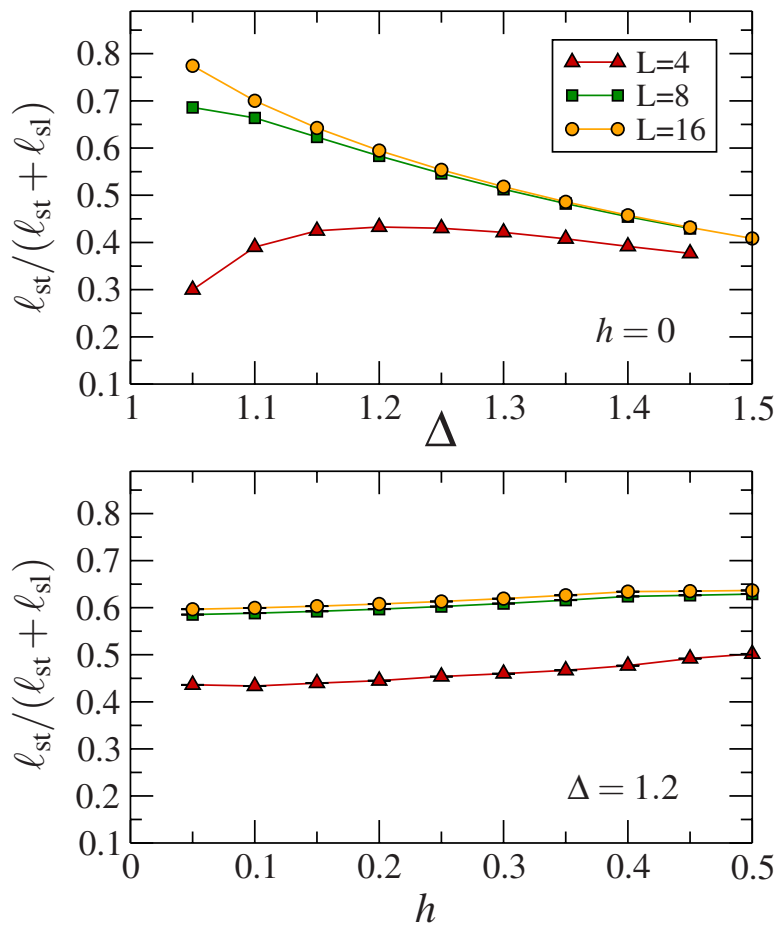

FIG. 5. (Color online) Tail length as a fraction of the total cluster size, measured in a number of vertices visited, for simulations of the 2D $X X Z$ model employing the short-loop algorithm and one particular solution of the directed-loop equations [12]. Here, $\beta$ $=2 L$.

efficient execution of storage and propagation processes. CPU and memory requirements can vary considerably depending on this implementation. In the section below, we provide some quantification of the short-loop efficiency with one particular $\mathrm{C}++$ implementation, using standard STL data structures.

\section{SIMULATION RESULTS FOR THE $X X Z$ MODEL}

For concreteness, we present a comparison of the efficiency of the long- and short-loop algorithms in the simple $X X Z$ model, Eq. (3), where in the below data we have set $J=1$. One of the first important indicators of short-loop efficiency is the length of the dangling tail, i.e., the discarded list of vertices that are not included in the definition of the closed loop for the purposes of updating the simulation. Long tails are generally detrimental to loop efficiency, due to the wasted CPU effort in both constructing and erasing them. In Fig. 5, this ratio is illustrated for several simulation sizes and parameter values, where the ratio of the tail length $\left(\ell_{\mathrm{st}}\right)$ versus the total cluster size (tail plus loop, $\ell_{\mathrm{st}}+\ell_{\mathrm{sl}}$ ) is plotted. Here, the short-loop length $\left(\ell_{\mathrm{s} 1}\right)$ is defined without bounce or back-tracking processes (see Fig. 4). From this figure, it is clear that the ratio $\ell_{\mathrm{st}} / \ell_{\mathrm{sl}}$ depends highly on simulation parameters; however, one tends to see convergence in parameter regions of large $\Delta$ or $h$, particularly with system size. This demonstration shows that the tail in the short-loop algorithm is, for these parameters, on the order of the length of the loop itself. One would prefer perhaps the existence of shorter tails on average; however, we are careful to note here that, in many cases for the $X X Z$ model, the average retained loop length can be quite small (only several vertices). It would clearly be interesting to address this ratio in other, more complicated Hamiltonians, which tend to produce larger loops.

Other characterizations of the short loop are possible, in particular a comparison of the (retained) loop length to the long-loop length. In this case, several definitions of loop "length" are possible. For example, we might be interested in the short-loop $\ell_{\mathrm{sl}}$ mentioned above, compared to a "conventional" definition of the long-loop length $\ell_{11}$ (which does not account for bounces or backtracking such as in Fig. 4). Again, this comparison is expected to depend highly on the model, lattice size and dimension, and parameter region one is simulating. We find in this simple $X X Z$ model, for example, that the ratio $\ell_{11} / \ell_{\mathrm{sl}} \approx 5$ to 10 , for simulations with $h=0$ and $\Delta>1$ (depending on $L$ and other factors). For simulations with $\Delta=0$ and finite $h>0, \ell_{11} / \ell_{\mathrm{sl}}$ begins at approximately 20 and falls off as $h$ increases. Because we choose an equilibration condition that the number of vertices traversed be constant and equal (discussed more below), the ratio of the number of loops performed by the long loop to the small loop reflects very closely the inverse of the ratio $\ell_{11} / \ell_{\mathrm{sl}}$. Depending on implementation, the proportional amount of CPU time spent on the short-loop algorithm can also be a significant constant multiple of this ratio.

We turn to what is perhaps the most quantitative indicator of simulation performance-measurements of autocorrelation functions for observable parameters [13]. The autocorrelation for a Monte Carlo time series of observables $O(1)$, $O(2), \ldots$, is defined with the normalized correlation function,

$$
A[O](t)=\frac{\langle O(i+t) O(t)\rangle-\langle O(i)\rangle^{2}}{\left\langle O(i)^{2}\right\rangle},
$$

where the averages are over the Monte Carlo "time" steps $i$ (elements of the Markov chain). Higher autocorrelations imply that series elements are less independent, while small autocorrelation times are a necessary condition for a simulation to be ergodic in a specific region of configuration space.

Before we proceed, we caution that one must be careful to note that quantitative values of autocorrelation functions are highly dependent on several simulation variables, which might be most concisely summed up as the definition of a Monte Carlo step (MCS). The MCS defines the increment $i$ in the definition (7), and hence critically affects the measurement of this quantity. In the SSE QMC, a MCS is typically defined as a diagonal update (mentioned above), followed by a number of operator loops. Changes incurred within these updates are mapped onto the stored basis state $|\alpha\rangle$ and operator string $S_{M}$, at which point the MCS is completed (and subsequently repeated). The number of operator loops is perhaps the most important variable in the definition of a MCS, and upon consideration it immediately becomes clear that this number is potentially defined much differently in a long versus a short loop, since the loop length discussed above varies considerably between the two. For example, a typical [12] way to define a MCS is that it contains a number of 


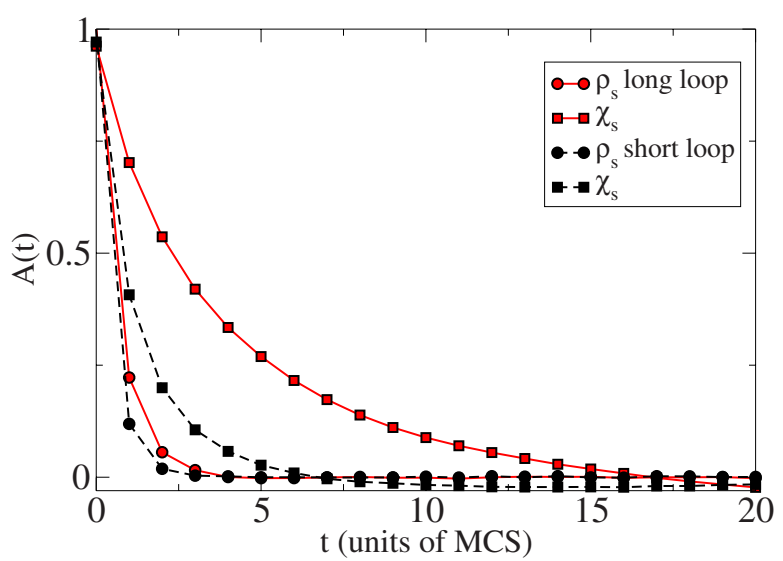

FIG. 6. (Color online) Autocorrelations of $\rho_{s}$ and $\chi_{s}$, plotted as a function of time in Monte Carlo steps, for the $X X Z$ model with $L$ $=16, \beta=32, h=0$, and $\Delta=1.1$ for both the long-loop (colored symbols) and short-loop (solid symbols) algorithms. Significant improvement of the autocorrelations for the short-loop over that for the long-loop algorithm is observed.

loops $\left(N_{\text {loop }}\right)$ that on average will traverse each vertex leg in the linked list one time,

$$
\bar{N}_{\text {leg }}=c_{l} N_{\text {loop }} \bar{\ell}_{\text {loop }},
$$

i.e., the constant $c_{l}$ is set to 1 . Here, $\bar{N}_{\text {leg }}$ is the number of vertices $(n)$ in the expansion multiplied by the number of legs per vertex (four for the simple $X X Z$ model), and the average loop length (the number of legs visited by each loop, $\left.\bar{\ell}_{\text {loop }}\right)$ may be approximated during equilibration time. A smaller $c_{l}$ will in general result in larger autocorrelations, since by definition each MCS traverses less vertex legs, resulting in more dependence between configurations in adjacent QMC steps. In the following results we set $c_{l}=0.25$, and adjust $N_{\text {loop }}$ during equilibration to satisfy Eq. (8). This value of $c_{l}$ is smaller than convention; however, it increases our autocorrelations to a manageable value in this simple model.

Another consideration not taken into account by simple autocorrelation function comparisons is the CPU effort involved when the definition of a MCS varies significantly, as in our case. Clearly, the short-loop algorithm involves both the overhead of storage of the stack data structure (containing both the loop and the tail), as well as the additional computational effort of erasing the tail at the end of loop construction. Indeed we observe that a short-loop MCS takes more CPU time than an analogously defined long-loop MCS, although the extent to which depends highly on algorithmic implementation and compiler optimization. Nonetheless, we keep this in mind in the following discussion.

Figure 6 illustrates autocorrelations versus Monte Carlo time step for two common observables, the spin stiffness $\rho_{s}$ [24] and the staggered susceptibility $\chi_{s}$ [18], for the $X X Z$ model with parameters $L=16, h=0$, and $\Delta=1$.1. It is already apparent that the short loop considerably improves autocorrelations for both observables in this simple demonstration. The CPU time involved in the short-loop run was larger than the long-loop run by a factor of 4 in this case. Upon reflec-
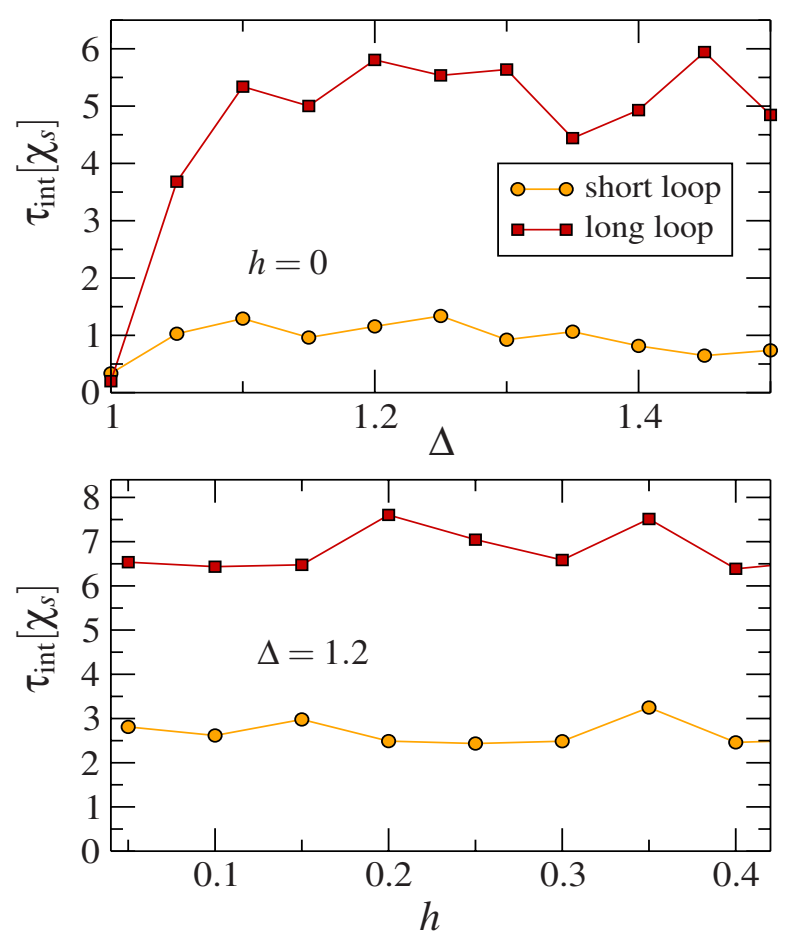

FIG. 7. (Color online) Integrated autocorrelation time for a $L$ $=16$ system at $\beta=32$. Error bars, although not plotted explicitly, are evident in the magnitude of fluctuations of the data points.

tion however, it is perhaps remarkable that the short loop gives any improvements to autocorrelations whatsoever, since presumably [as chosen by the equilibrium condition (8)] the same average number of vertex legs have been traversed by both algorithms. This is possibly a measure of the degree to which the elimination of retracing (Fig. 3), or the flipping of many independent short loops (discussed previously), gives efficiency improvements over the long-loop algorithm. It would clearly be interesting to study this issue in more detail in the future.

We concentrate now on autocorrelation measurements of the slowest decay in Fig. 6, the staggered spin susceptibility. To further characterize algorithmic efficiency, we look at integrated autocorrelation times, defined as

$$
\tau_{\text {int }}[O]=\frac{1}{2}+\sum_{t=1}^{\infty} A[O](t),
$$

using $O=\chi_{s}$. Figure 7 illustrates this calculation for short versus long loops as one sweeps in parameters $\Delta$ and $h$, for $L=16$, using one particular solution to the directed-loop equations which minimize bounces [12]. As evident from Fig. 8, this system size is large enough that any remaining finite-size effects are obscured by statistical errors (note also that the computational effort of the SSE QMC scales linearly in both $\beta$ and $L$ ). The quantitative value of $\tau_{\text {int }}\left[\chi_{s}\right]$ depends highly on the definition of the MCS $\left(c_{l}\right)$, as well as the directed-loop equations [using a heat-bath solution [12] increases $\tau_{\text {int }}\left[\chi_{s}\right]$ considerably]. In some cases, for example, in certain bounce-free regions of parameter space, integrated 


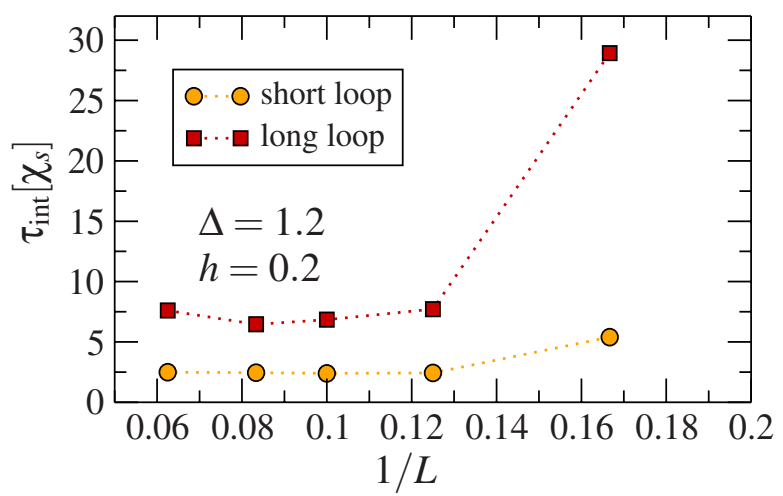

FIG. 8. (Color online) Integrated autocorrelation time for simulations with $\Delta=1.2$ and $h=0.2$, versus the inverse linear system size. The inverse temperature of each run is set at $\beta=2 L$. Error bars are roughly equivalent to the symbol size.

autocorrelations are very small and the difference between short- and long-loop performance is lost in the statistical errors. However, for another large region of phase space (such as that illustrated in Fig. 7), the general trend is that the short-loop algorithm outperforms the long loops in terms of $\tau_{\text {int }}\left[\chi_{s}\right]$. Surprisingly, even in the simple $X X Z$ model, we were unable to find regions of parameter space where $\tau_{\text {int }}\left[\chi_{s}\right]$ is significantly larger for the short-loop algorithm than for the long-loop algorithm. That being said, we observe that in our implementations of the short-loop algorithm, the amount of CPU time required to produce results such as in Fig. 7 are significantly larger for the short-loop code, typically by a factor of 4 or more. Memory allocation is also larger for the short loops, although as with most QMC simulations still relatively small compared to hardware constraints, offering no real disadvantage over the conventional long-loop algorithm.

Recall, the purpose of the short loop is perhaps not to bring significant efficiency gains to all models, rather to those models where long-loop length tends to get excessive, causing in some cases the loop to be truncated. Figure 9 demonstrates the fact that this practice of truncating long loops in the SSE QMC results in a systematic increase in the integrated autocorrelation time. By comparison, the same set of parameters using the short-loop algorithm results in a $\tau_{\text {int }}\left[\chi_{s}\right] \approx 1$, when run using the same condition $\left[c_{l}=0.25\right.$ in Eq. (8)] to define the QMC (with a smaller number of short loops, this value will increase). Thus, it is clear that the advantage in using the short-loop algorithm will only increase in events where long loops are observed to become aborted (or excessively long), such as those expected to occur on more complicated quantum models.

\section{DISCUSSION}

Motivated by the success of short-loop algorithms in Monte Carlo simulations of classical vertex and ice models, we have presented an adaptation of the short-loop algorithm for use in QMC simulations of quantum lattice Hamiltonians. This quantum short-loop algorithm is a modification of the

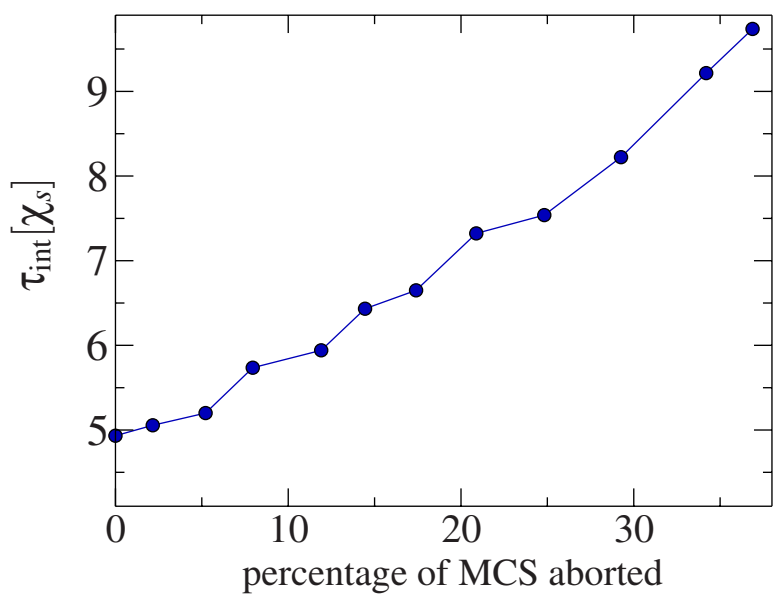

FIG. 9. (Color online) The integrated autocorrelation time for a $L=8, \beta=16$ system with $h=0$ and $\Delta=1.4$. The $x$ axis is the percentage of Monte Carlo steps that are aborted due to the termination of a long loop. This truncation percentage is controlled by restricting the maximum loop length with Eq. (6).

conventional ("long") quantum loop or worm algorithm, whereby smaller clusters in the $d+1$-dimensional simulation cell may be constructed and updated. Short loops are defined by a modified construction algorithm, where a propagating defect closes a loop upon crossing a part of its previously visited path. Unlike the conventional long loop, this results in a dangling tail that must be removed before the QMC algorithm can continue. Within the SSE QMC framework, we introduced the general algorithmic rules and data structures required for constructing and updating short loops (including an additional stack to store the loop under construction), and outlined some expected advantages and disadvantages of the new algorithm as compared to the conventional long-loop algorithm.

Using a $\mathrm{C}++$ implementation of the short-loop algorithm in the simple square lattice $S=1 / 2 X X Z$ model, we characterized key aspects of simulation performance, and compared to the conventional long-loop algorithm using identical parameters. Remarkably, the short-loop algorithm is observed to give much smaller autocorrelation times-a key indicator that this modification results in an increase in simulation efficiency. However, with this improvement in autocorrelation time comes a significant increase in CPU effort (and to a lesser extent memory usage). Hence, before using the shortloop algorithm in large-scale QMC simulations, one must be careful in identifying models and parameter regions where this compromise becomes favorable.

Significant work still remains to be done in optimizing and characterizing the short-loop algorithm, particularly in different QMC flavors and on more complicated quantum models. Although conventional estimators such as those discussed here will remain unbiased by the short-loop algorithm, it remains to be determined whether certain schemes to measure Green's functions and dynamical properties are affected by the smaller loops that are generated [25]. In the immediate future, the quantum short-loop algorithm will likely be most useful in specific complicated models (e.g., 
those with long-range interactions in the Hamiltonian) where conventional long loops are observed to behave poorly, rather than as a means of improving efficiency in the general case. Eventually, a more wide-spread adoption of the short loop may be warranted, although further implementation and characterization on additional models will be required to more clearly identify its strengths and weaknesses.

\section{ACKNOWLEDGMENTS}

The authors thank A. Sandvik, M. Troyer, and S. Wessel for valuable discussions. The authors thank the hospitality of
KITP, Santa Barbara, where part of this work was done. One of the authors (Y.J.K.) thanks the National Center for High Performance Computing of Taiwan, and the Computer and Information Networking Center at NTU for the support of high-performance computing facilities. One of the authors (R.G.M.) acknowledges use of the facilities of the Shared Hierarchical Academic Research Computing Network (SHARCNET). This work was partly supported by the National Center for Theoretical Sciences and NSC of Taiwan under Grant No. 96-2112-M-002-010, (Y.J.K.), NSF under Grant No. PHY05-51164 (Y.J.K. and R.G.M.), and the U.S. Department of Energy Contract No. DE-AC05-00OR22725 with ORNL, managed by UT-Battelle, LLC (R.G.M.).
[1] M. Suzuki, Prog. Theor. Phys. 56, 1454 (1976).

[2] D. C. Handscomb, Proc. Cambridge Philos. Soc. 58, 594 (1962).

[3] A. W. Sandvik, S. Daul, R. R. P. Singh, and D. J. Scalapino, Phys. Rev. Lett. 89, 247201 (2002).

[4] See, for example, O. Gygi, H. G. Katzgraber, M. Troyer, S. Wessel, and G. G. Batrouni, Phys. Rev. A 73, 063606 (2006), and references therein.

[5] E. Y. Loh, J. E. Gubernatis, R. T. Scalettar, S. R. White, D. J. Scalapino, and R. L. Sugar, Phys. Rev. B 41, 9301 (1990).

[6] M. Troyer and U.-J. Wiese, Phys. Rev. Lett. 94, 170201 (2005).

[7] N. Kawashima and K. Harada, J. Phys. Soc. Jpn. 73, 1379 (2004).

[8] H. G. Evertz, G. Lana, and M. Marcu, Phys. Rev. Lett. 70, 875 (1993).

[9] N. V. Prokof'ev, B. V. Svistunov, and I. S. Tupitsyn, J. Exp. Theor. Phys. 87, 310 (1998).

[10] M. Boninsegni, Nikolay Prokof'ev, and B. Svistunov, Phys. Rev. Lett. 96, 070601 (2006).

[11] A. W. Sandvik, Phys. Rev. B 59, R14157 (1999).

[12] O. F. Syljuåsen and A. W. Sandvik, Phys. Rev. E 66, 046701 (2002).
[13] J. S. Liu, Monte Carlo Strategies in Scientific Computing (Springer, New York, 2001).

[14] A. Rahman and F. Stillinger, J. Chem. Phys. 57, 4009 (1972).

[15] G. T. Barkema and M. E. J. Newman, Phys. Rev. E 57, 1155 (1998).

[16] N. Prokof'ev and B. Svistunov, Phys. Rev. Lett. 87, 160601 (2001).

[17] R. G. Melko and M. J. P. Gingras, J. Phys.: Condens. Matter 16, R1277 (2004).

[18] A. W. Sandvik and J. Kurkijarvi, Phys. Rev. B 43, 5950 (1991).

[19] A. W. Sandvik, J. Phys. A 25, 3667 (1992).

[20] A. W. Sandvik (private communication).

[21] P. Sengupta, A. W. Sandvik, and D. K. Campbell, Phys. Rev. B 65, 155113 (2002).

[22] F. Alet, S. Wessel, and M. Troyer, Phys. Rev. E 71, 036706 (2005).

[23] L. Pollet, S. M. A. Rombouts, K. Van Houcke, and K. Heyde, Phys. Rev. E 70, 056705 (2004).

[24] E. L. Pollock and D. M. Ceperley, Phys. Rev. B 36, 8343 (1987).

[25] A. Dorneich and M. Troyer, Phys. Rev. E 64, 066701 (2001). 